\title{
In vivo free-breathing DTI \& IVIM of the whole human heart using a real-time slice-followed SE- EPI navigator-based sequence: a reproducibility study in healthy volunteers
}

Kévin Moulin ${ }^{2,1^{*}}$, Pierre Croisille ${ }^{2,4}$, Thorsten Feiweier ${ }^{3}$, Benedicte M Delattre ${ }^{2}$, Hongjiang Wei $^{2}$, Benjamin Robert ${ }^{1}$, Olivier Beuf², Magalie Viallon 2,4

From 18th Annual SCMR Scientific Sessions

Nice, France. 4-7 February 2015

\section{Background}

In vivo cardiac diffusion using either the Intra-Voxel Incoherent Motion (IVIM) model or the Diffusion Tensor Imaging (DTI) model shows promise to provide new insights into cardiac disease processes. However, due to the combined challenge of respiratory and heart motion $[1,2]$, currently proposed acquisition methods may not be applicable in patients due to long acquistion time. In this study, we propose to use the motion information provided by a navigator to prospectively update in real time the position of the diffusion weighted slices, offering an efficient free-breathing strategy for rapid and improved cardiac diffusion acquisition using a singleshot spin-Echo EPI sequence.

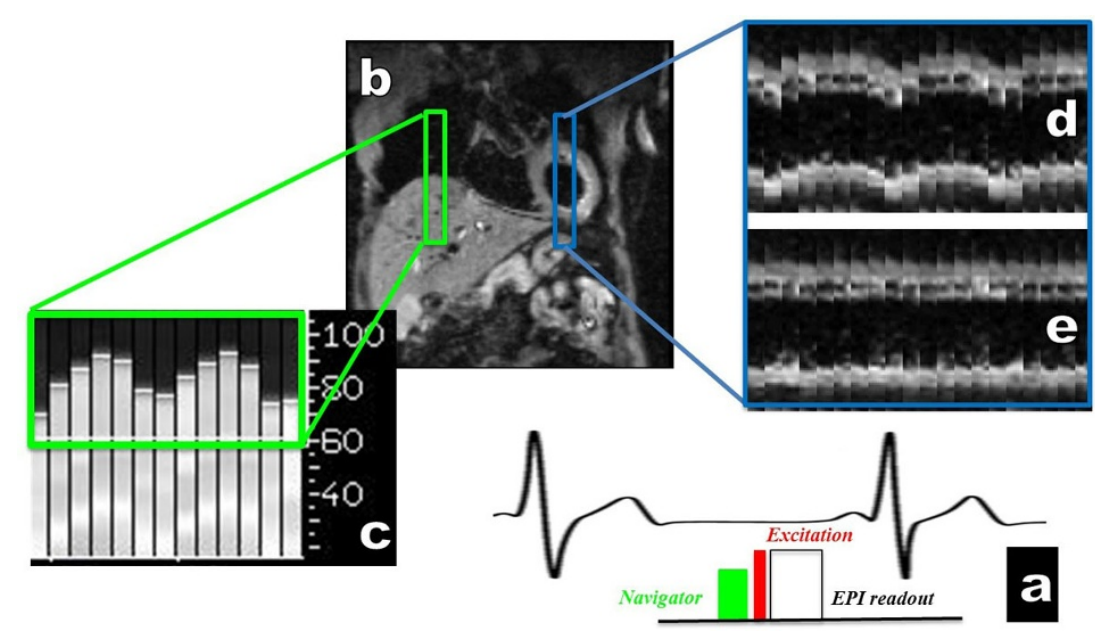

Figure 1 a) A pencil beam navigator is played out before each acquisition and placed on the top of the liver b). The information given by the navigator $c$ ) is used, through a tracking factor of 0.6 , to move the slice in real time during the breathing cycle.

Performance of the respiratory head-foot motion correction for SE-EPI diffusion with slice following was evaluated with coronal b-values=30 s/ mm2 image from a series of diffusion-weighted images acquired over $1 \mathrm{~min}$. d) and e) breathing coronal acquisition without and with slice following.

${ }^{2}$ CREATIS; CNRS (UMR 5220); INSERM (U1044); INSA Lyon; Université de Lyon,

Lyon, France

Full list of author information is available at the end of the article 


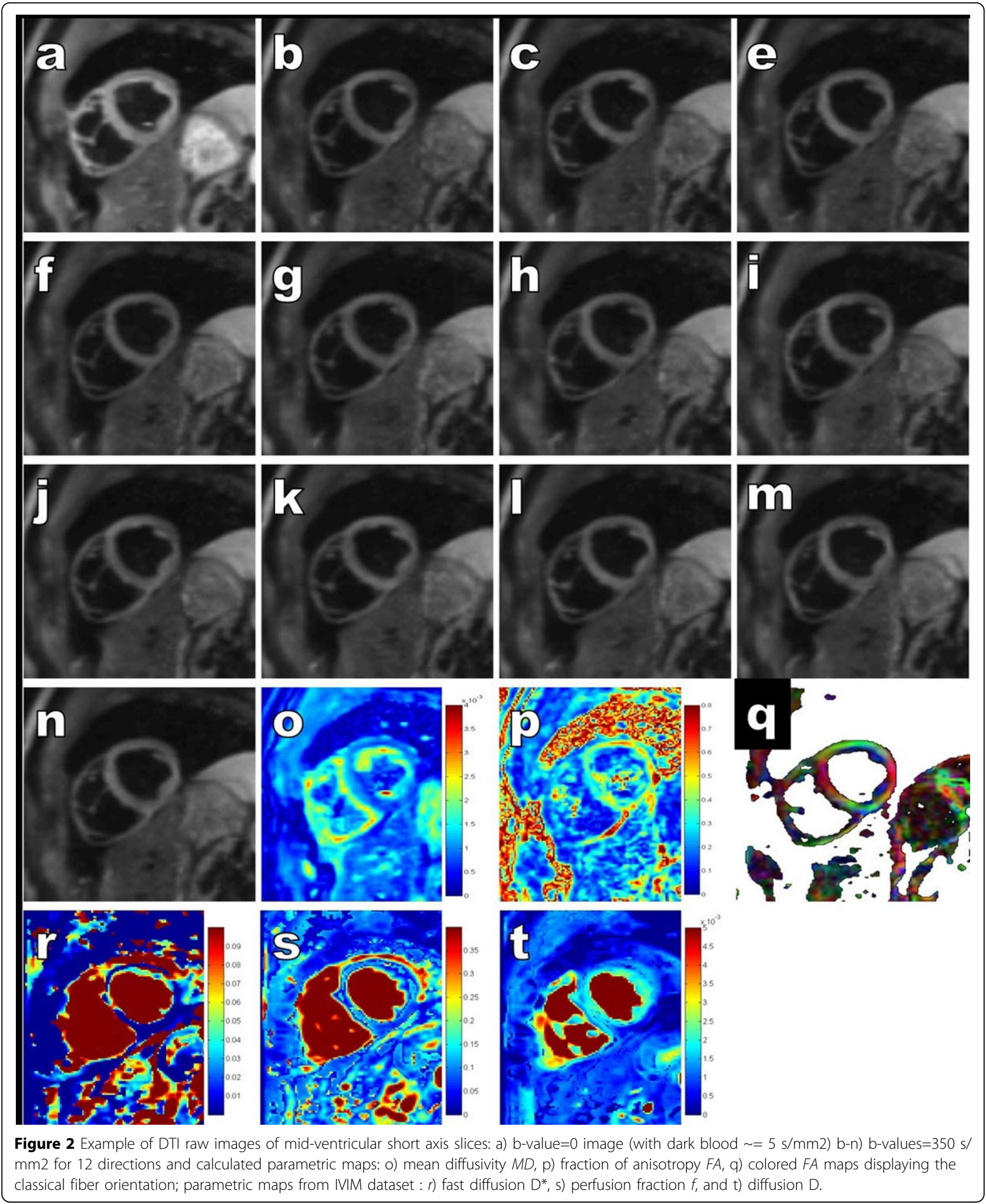

\section{Methods}

The proposed technique was performed on 10 volunteers on $1.5 \mathrm{~T}$ scanner to quantify the reproducibility of the diffusion parameters $(F A, M D, f, D$ and $D *)$. Double oblique short-axis DW images were acquired with a 128 $\times 80$ pixel matrix and rectangular FoV of $350 \times$ 
220mm2. Short axis DW-slices (6-mm-thick) were obtained with an in-plane resolution of $2.7 \times 2.7 \mathrm{~mm} 2$ interpolated in-plane to $1.35 \times 1.35 \mathrm{~mm} 2$; the bandwidth was $2442 \mathrm{~Hz} /$ pixel, and GRAPPA factor 2 was used. Multiple TDs shifted every $10 \mathrm{~ms}$ were acquired for PCAtMIP reconstruction. DTI acquisitions parameters were: interleaved 10 contiguous slices; $\mathrm{TE}=45 \mathrm{~ms}$; $\mathrm{TR}=$ number of slices $x$ heart rate (e.g., 10s for a heart rate equal to $60 \mathrm{bpm}$ ); and 12 diffusion directions with a bvalue of $350 \mathrm{~s} / \mathrm{mm} 2$ and 10 TDs for a total acquisition time of $22 \mathrm{~min}$. The IVIM acquisition parameters were 5 interleaved slices with a gap of $100 \%$; $\mathrm{TE}=42 \mathrm{~ms}$; $\mathrm{TR}=5 \mathrm{~s}$; 6 diffusion directions with b-values $0,15,30,50,75$, $100,200,300,400 \mathrm{~s} / \mathrm{mm} 2$ and 5 TDs were acquired for total time of $20 \mathrm{~min}$. Inter-measurement reproducibility among repeated measures was assessed by the Lin concordance coefficient (LinCC) and the complementary Bland and Altman method with LinCC coefficient $\rho_{c}, r$ (a measure of precision) and $C_{b}$ (a measure of accuracy).

\section{Results}

With a $100 \%$ breathing cycle scanning efficiency, the slicefollowing strategy is a powerful head-foot respiratory motion management solution for SE-EPI cardiac diffusion Vascular fraction $f$ and the diffusion coefficients $D$ and $D^{*}$ were determined to be $35.6 \pm 11.9 \times 10^{-3} \mathrm{~mm} 2 / \mathrm{s}$ for $D^{*}, 1.23$ $\pm 0.19 \times 10^{-3} \mathrm{~mm} 2 / \mathrm{s}$ for $D$ and $0.115 \pm 0.017$ for $f$. From the DTI protocol, the Mean Diffusivity $(M D)$ was 1.57 $\pm 0.13 \times 10^{-3} \mathrm{~mm} 2 / \mathrm{s}$ and the Fractional Anisotropy $(F A) 0.35$ $\pm 0.04 . M D\left(\rho_{c}=0.69, r=0.70, C_{b}=0.99\right), D\left(\rho_{c}=0.92, r=0.99\right.$, $\left.C_{b}=0.93\right)$ and $f\left(\rho_{c}=0.69, r=0.75, C_{b}=0.91\right)$ was highly reproducible exept for $D^{*}\left(\rho_{c}=-0.214, r=-0.229, C_{b}=0.93\right)$ and $F A$ $\left(\rho_{c}=0.075, r=0.12, C_{b}=0.56\right)$ but which remained accurate.

\section{Conclusions}

The slice-followed SE-EPI cardiac diffusion sequence is a promising solution for clinical implementation, allowing unprecedent acquisition speed that could be tailored to specific needs.

\section{Funding}

N/A.

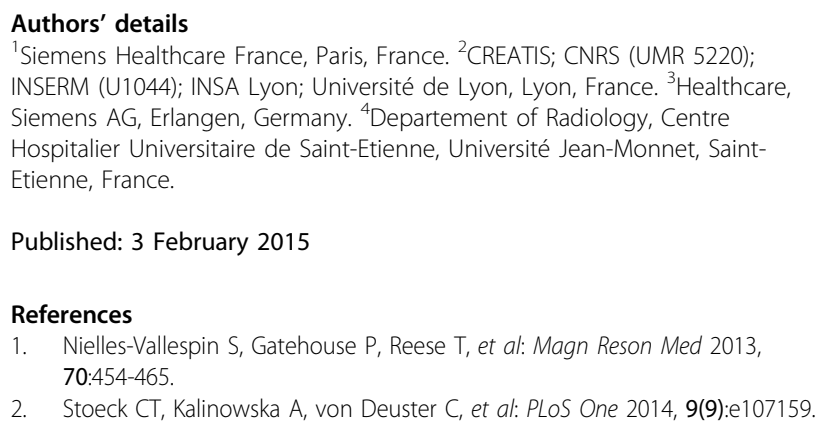

Published: 3 February 2015

\section{References}

1. Nielles-Vallespin S, Gatehouse P, Reese T, et al: Magn Reson Med 2013, 70:454-465.

2. Stoeck CT, Kalinowska A, von Deuster C, et al: PLoS One 2014, 9(9):e107159.

doi:10.1186/1532-429X-17-S1-P383

Cite this article as: Moulin et al:: In vivo free-breathing DTI \& IVIM of the whole human heart using a real-time slice-followed SE-EPI navigator-based sequence: a reproducibility study in healthy volunteers. Journal of Cardiovascular Magnetic Resonance 2015 17(Suppl 1):P383.
Submit your next manuscript to BioMed Central and take full advantage of:

- Convenient online submission

- Thorough peer review

- No space constraints or color figure charges

- Immediate publication on acceptance

- Inclusion in PubMed, CAS, Scopus and Google Scholar

- Research which is freely available for redistribution
C Biomed Central 